\title{
Simulating Hydrodynamics on Noisy Intermediate-Scale Quantum Devices with Random Circuits
}

\author{
Jonas Richter( $\odot^{*}$ and Arijeet Pal \\ Department of Physics and Astronomy, University College London, \\ Gower Street, London WC1E 6BT, United Kingdom
}

(Received 16 December 2020; accepted 14 May 2021; published 8 June 2021)

\begin{abstract}
In a recent milestone experiment, Google's processor Sycamore heralded the era of "quantum supremacy" by sampling from the output of (pseudo-)random circuits. We show that such random circuits provide tailor-made building blocks for simulating quantum many-body systems on noisy intermediate-scale quantum (NISQ) devices. Specifically, we propose an algorithm consisting of a random circuit followed by a trotterized Hamiltonian time evolution to study hydrodynamics and to extract transport coefficients in the linear response regime. We numerically demonstrate the algorithm by simulating the buildup of spatiotemporal correlation functions in one- and two-dimensional quantum spin systems, where we particularly scrutinize the inevitable impact of errors present in any realistic implementation. Importantly, we find that the hydrodynamic scaling of the correlations is highly robust with respect to the size of the Trotter step, which opens the door to reach nontrivial time scales with a small number of gates. While errors within the random circuit are shown to be irrelevant, we furthermore unveil that meaningful results can be obtained for noisy time evolutions with error rates achievable on near-term hardware. Our work emphasizes the practical relevance of random circuits on NISQ devices beyond the abstract sampling task.
\end{abstract}

DOI: 10.1103/PhysRevLett.126.230501

Introduction.-Studying the properties of quantum many-body systems is tremendously challenging [1]. Notwithstanding significant progress thanks to the development of sophisticated numerical methods [2-7] and groundbreaking experiments with cold-atom or trappedion platforms [8,9], simulations on universal quantum computers promise to yield major advancements in a multitude of research areas [10,11]. While a fault-tolerant quantum computer is still far into the future, noisy intermediate-scale quantum (NISQ) devices are available and their current capabilities have been demonstrated for various problems such as electronic structure calculations $[12,13]$, simulations of spectral functions [14,15], measurement of entanglement $[16,17]$, topological phase transitions [18], and out-of-equilibrium dynamics [19-22].

Recently, an important milestone toward so-called "quantum supremacy" [23] has been achieved by using Google's NISQ device Sycamore [24]. In the experiment, the Josephson junction-based quantum processor was used to sample from the output distribution of (pseudo-)random circuits involving up to 53 qubits, thereby going beyond the capacities of modern supercomputers. As this sampling task may appear rather abstract, it is crucial to identify a wider range of relevant applications of near-term NISQ devices that can be performed despite their imperfect fidelities of one- and two-qubit gates and the lack of error correction [25-28].
Transport processes represent one of the most generic nonequilibrium situations [29]. In the quantum realm, the understanding of transport not only plays a key role to pave the way for future technologies such as spintronics [30], but is also intimately related to fundamental questions of equilibration and thermalization in many-body systems [31-33]. While quantum transport has been experimentally studied in mesoscopic systems, solid-state quantum magnets, and cold-atom settings (see, e.g., [34-38]), active questions from the theory side include the quantitative calculation of transport coefficients [29,39], as well as explaining the emergence of conventional hydrodynamic transport from the underlying unitary time evolution of closed quantum systems [40].

In this Letter, we advocate near-term NISQ devices as useful platforms for simulating hydrodynamics in quantum many-body systems and, in particular, we show that random circuits (as realized in [24]) form tailor-made building blocks for this purpose. With generalizations being possible [41] (see also Supplemental Material [42]), we specifically propose an efficient scheme to compute the infinite-temperature spatiotemporal correlation function $C_{\ell, \ell^{\prime}}(t)$ for one- and two-dimensional (1D, 2D) quantum spin systems,

$$
C_{\ell, \ell^{\prime}}(t)=\operatorname{Tr}\left[S_{\ell}^{z}(t) S_{\ell^{\prime}}^{z}\right] / 2^{L},
$$


where $S_{\ell\left(\ell^{\prime}\right)}^{z}$ is a spin-1/2 operator at lattice site $\ell\left(\ell^{\prime}\right)$, $S_{\ell}^{z}(t)=e^{i \mathcal{H} t} S_{\ell} e^{-i \mathcal{H} t}$ is the time-evolved operator with respect to some Hamiltonian $\mathcal{H}$, and $L$ denotes the number of spins (qubits). The spatiotemporal correlations $C_{\ell, \ell^{\prime}}(t)$ are central objects for studying transport within linear response theory [29], as well as thermalization and manybody localization in quantum systems [43]. As a key ingredient, our scheme exploits the concept of quantum typicality [44-46], which asserts that ensemble averages can be accurately approximated by an expectation value with respect to a single pure state drawn at random from a high-dimensional Hilbert space [47-49]. Remarkably, typicality applies independent of concepts such as the eigenstate thermalization hypothesis [31] and remains valid also for integrable or many-body localized systems [50].

While random pure states have a long history for efficient numerical simulations [50-59], we demonstrate in this Letter that typicality can be used to recast the correlation function $C_{\ell, \ell^{\prime}}(t)$ into a form that can be readily evaluated on a quantum computer (see [42] for a derivation),

$$
C_{\ell, \ell^{\prime}}(t)=\frac{1}{2}\left\langle\psi_{\mathcal{R}, \ell^{\prime}}(t)\left|S_{\ell}^{z}\right| \psi_{\mathcal{R}, \ell^{\prime}}(t)\right\rangle+\mathcal{O}\left(2^{-L / 2}\right),
$$

where $\left|\psi_{\mathcal{R}, \ell^{\prime}}(t)\right\rangle=e^{-i \mathcal{H} t}\left|\psi_{\mathcal{R}, \ell^{\prime}}\right\rangle$, and $\left|\psi_{\mathcal{R}, \ell^{\prime}}\right\rangle=|0\rangle_{\ell^{\prime}} \otimes$ $\mathcal{R}|0\rangle^{\otimes L-1}$ results from the application of a (pseudo-) random circuit $\mathcal{R}$ on all qubits of the system except for the fixed reference site $\ell^{\prime}$. Importantly, as indicated by the second term on the right-hand side (rhs), the accuracy of Eq. (2) improves exponentially with the size of the system [58]. Complementary to well-known approaches to obtain correlation functions such as Eq. (1) on a quantum computer [60-62] (see also [63]), the scheme proposed in this Letter operates without requiring an overhead of bath or ancilla qubits for initial-state preparation and measurement. Rather, it combines the random-circuit technology already realized on NISQ devices [24] with "quantum parallelism" [53,64], as the time evolution of a single random state $\left|\psi_{\mathcal{R}, \ell^{\prime}}\right\rangle$ suffices to capture the full ensemble average (1). Furthermore, we particularly scrutinize the impact of Trotter and gate errors present in any realistic implementation and discuss the possibility to extract transport coefficients with error rates achievable on near-term hardware.

Description of setup.-First, all qubits are initialized in the $|0\rangle$ state. The algorithm then consists of a random circuit $\mathcal{R}$ acting on $L-1$ qubits followed by a time evolution $U(t)$ on all $L$ sites [Fig. 1(a)]. $\mathcal{R}$ comprises individual cycles, each composed of a layer of one-qubit gates and a layer of twoqubit gates, with $d$ denoting the total number of cycles [Fig. 1(b)]. In each cycle, the one-qubit gates are randomly chosen from the set $\left\{X^{1 / 2}, Y^{1 / 2}, T\right\}$, where $X^{1 / 2}\left(Y^{1 / 2}\right)$ are $\pi / 2$ rotations around the $x$ axis ( $y$ axis) of the Bloch sphere and $T$ is the non-Clifford gate $T=\operatorname{diag}\left(1, e^{i \pi / 4}\right)$.

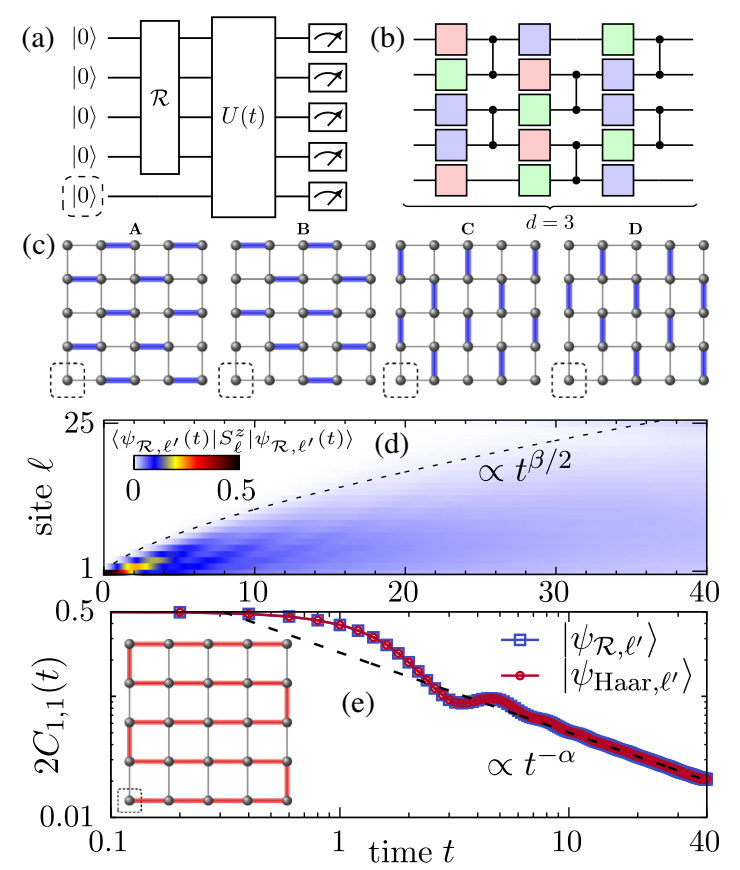

FIG. 1. (a) A random circuit $\mathcal{R}$ acts on $L-1$ qubits, followed by a time evolution $U(t)$ on all $L$ sites. (b),(c) $\mathcal{R}$ comprises $d$ cycles, each composed of layers of one- and two-qubit gates. We consider a two-dimensional geometry and $\mathbf{A}-\mathbf{D}$ are patterns of two-qubit gates used in different cycles. (d),(e) For reference site $\ell^{\prime}=1,\left\langle\psi_{\mathcal{R}, \ell^{\prime}}(t)\left|S_{\ell}^{z}\right| \psi_{\mathcal{R}, \ell^{\prime}}(t)\right\rangle$ yields the correlation function $2 C_{\ell, 1}(t)$. Data are shown for the spin-1/2 Heisenberg chain with $L=25$, where the one-dimensional system is realized as a snakelike path through the lattice. Panel (e) shows a cut at $\ell=1$. Even for shallow $\mathcal{R}$ with $d=20$, results from $\left|\psi_{\mathcal{R}, \ell^{\prime}}\right\rangle$ are indistinguishable from a true Haar-random state. Dashed lines indicate power-law scalings of the correlations; cf. Eq. (6).

We impose the constraint that the one-qubit gates on a given site have to be different in two subsequent cycles. As a two-qubit gate, we consider the controlled-Z (CZ) gate, $\mathrm{CZ}=\operatorname{diag}(1,1,1-1)$. (See [42] for circuits with CNOT gates.) In each cycle, the $\mathrm{CZ}$ gates are aligned in one of the patterns $\mathbf{A}-\mathbf{D}$ on a 2D geometry [Fig. 1(c)], where we repeat the sequence $\mathbf{A B C D} \ldots$ throughout $\mathcal{R}$, similar to Refs. [23,24]. After $d$ cycles, the state $\left|\psi_{\mathcal{R}, \ell^{\prime}}\right\rangle=\sum_{k} c_{k}|k\rangle$ is a superposition of computational basis states. It is the important realization that states generated from (shallow) random circuits $\mathcal{R}$ can approximate the properties of a Haar-random state $[23,65,66]$; i.e., the coefficients $c_{k}$ are expected to closely follow a Gaussian distribution with zero mean. (Note that the exact preparation of a Haarrandom state would be extremely inefficient in contrast [67].)

For the subsequent time evolution, we exemplarily consider the 1D and 2D spin-1/2 Heisenberg model with nearest-neighbor interactions (see [42] for results on another model [68]), where we identify $|0\rangle=|\uparrow\rangle$ and $|1\rangle=|\downarrow\rangle$, 


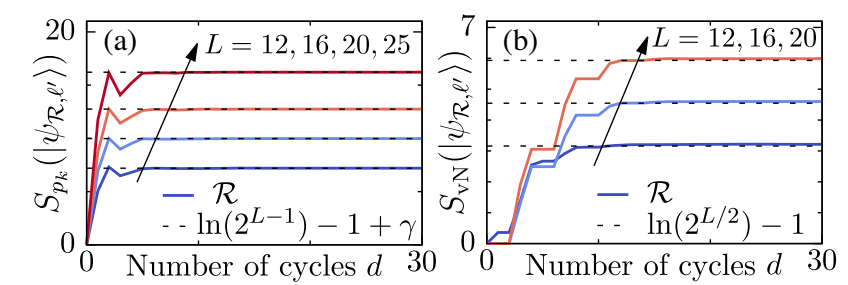

FIG. 2. Buildup of randomness of $\left|\psi_{\mathcal{R}, \ell^{\prime}}\right\rangle$. (a) $S_{p_{k}}\left(\left|\psi_{\mathcal{R}, \ell^{\prime}}\right\rangle\right)$ reaches the random-state value $\ln \left(2^{L-1}\right)-1+\gamma$ with Euler constant $\gamma \approx 0.577$ already at moderate $d$ [23]. (b) $S_{\mathrm{vN}}\left(\left|\psi_{\mathcal{R}, \ell^{\prime}}\right\rangle\right)$ approaches the "Page value" $\ln \left(2^{L / 2}\right)-1$ [71] appropriate for a random state on $L-1$ sites. The displayed $L$ values correspond to the $2 \mathrm{D}$ geometries $4 \times 3,4 \times 4,5 \times 4$, and $5 \times 5$. Data are averaged over 100 realizations of $\mathcal{R}$.

$\mathcal{H}=\sum_{\left\langle\ell, \ell^{\prime}\right\rangle} h_{\ell, \ell^{\prime}}=\sum_{\left\langle\ell, \ell^{\prime}\right\rangle} \mathbf{S}_{\ell} \cdot \mathbf{S}_{\ell^{\prime}}, \quad \mathbf{S}_{\ell}=\left(S_{\ell}^{x}, S_{\ell}^{y}, S_{\ell}^{z}\right)$,

where the $1 \mathrm{D}$ model is realized as a path through the $2 \mathrm{D}$ lattice [Fig. 1(e)]. Focusing (for now) on 1D, the timeevolution operator $U(t)=\exp (-i \mathcal{H} t)$ is Trotterized [69],

$$
U(t)=\left(e^{-i \mathcal{H} \delta t}\right)^{N} \approx\left(e^{-i \mathcal{H}_{\mathrm{e}} \delta t} e^{-i \mathcal{H}_{\mathrm{o}} \delta t}\right)^{N}+\mathcal{O}\left(\delta t^{2}\right),
$$

where $\mathcal{H}_{\mathrm{e}}\left(\mathcal{H}_{\mathrm{o}}\right)$ denotes the even (odd) bonds $h_{\ell, \ell^{\prime}}$ of $\mathcal{H}$, and $\delta t=t / N$ is a discrete time step. The mutually commuting two-site terms $\exp \left(-i h_{\ell, \ell^{\prime}} \delta t\right)$ are then translated into elementary one- and two-qubit gates [11]. (We here use a representation that requires three CNOT gates $[20,42,70]$.) Eventually, according to quantum typicality and our construction (see [42]), a $z$-basis measurement of the qubit at site $\ell$ after time $t$ then yields the correlation function $2 C_{\ell, \ell^{\prime}}(t)$ [Figs. 1(d) and 1(e)]. In particular, we show below that the correct extraction of $C_{\ell, \ell^{\prime}}(t)$ remains possible even in the presence of inevitable Trotter and gate errors.

Buildup of randomness. - In Fig. 2(a), we study the growth of $S_{p_{k}}\left(\left|\psi_{\mathcal{R}, \ell^{\prime}}\right\rangle\right)=-\sum_{k=1}^{2^{L}} p_{k} \ln p_{k}$ with $p_{k}=\left|c_{k}\right|^{2}$, which measures the spreading of $\left|\psi_{\mathcal{R}, \ell^{\prime}}\right\rangle$ within the computational basis due to $\mathcal{R}$. Moreover, the corresponding entanglement of $\left|\psi_{\mathcal{R}, \ell^{\prime}}\right\rangle$ is analyzed in Fig. 2(b) by means of the von Neumann entropy $S_{\mathrm{vN}}\left(\left|\psi_{\mathcal{R}, \ell^{\prime}}\right\rangle\right)=-\operatorname{Tr}\left[\rho_{A} \ln \rho_{A}\right]$, with $\rho_{A}=\operatorname{Tr}_{B}\left|\psi_{\mathcal{R}, \ell^{\prime}}\right\rangle\left\langle\psi_{\mathcal{R}, \ell^{\prime}}\right|$ being the reduced density matrix for a half-system bipartition into regions $A$ and $B$. Importantly, we observe that both $S_{p_{k}}$ and $S_{\mathrm{vN}}$ reach their theoretically expected values for a random state [23,71] already at moderate numbers of cycles $d \lesssim 10$, where the required $d$ appears to exhibit only a minor dependence on $L$ [23]. We thus expect that $\left|\psi_{\mathcal{R}, \ell^{\prime}}\right\rangle$ mimics a true Haarrandom state even for shallow $\mathcal{R}$ and can be used within the typicality approach to obtain $C_{\ell, \ell^{\prime}}(t)$. Throughout this Letter, we use a fixed value $d=20$, which yields very accurate results; see Fig. 1(e) and [42]. (Note that $d=20$ has already been realized for 53 qubits [24].) Eventually,

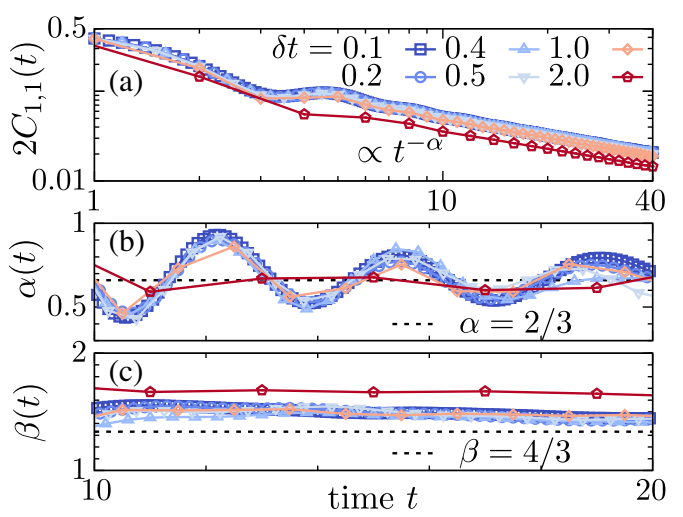

FIG. 3. Impact of the Trotter time step. (a) $C_{1,1}(t)$ for varying values of $\delta t$. (b),(c) Extracted power-law exponents $\alpha(t)$ and $\beta(t)$. The dashed lines indicate the KPZ scaling [29,76-81]. Data are obtained for $L=25$ and $d=20$.

we stress that this accuracy is achieved even though our design of $\mathcal{R}$ is not optimized [72,73]; i.e., no particular finetuning of $\mathcal{R}$ appears to be necessary.

Dependence on Trotter time step.-Given the eponymous noise of NISQ devices, it is desirable to use as few gates as possible, i.e., a large time step $\delta t$. However, for a larger $\delta t$, the systematic error of the Trotter decomposition is in turn expected to increase [see rhs of Eq. (4)]. In Fig. 3, we demonstrate that this expectation does not need to hold in practice (see Refs. $[74,75]$ ), such that a favorable trade-off between large $\delta t$ and acceptable Trotter error can be achieved. Specifically, we find that the equal-site correlation function $C_{1,1}(t)$ in Fig. 3(a) remains almost unchanged for varying $\delta t$ between $\delta t=0.1$ and $\delta t=1$. Even though small deviations appear for larger $\delta t=2$, the qualitative shape of $C_{1,1}(t)$ remains similar also in this case. For a more detailed analysis, we consider the emerging hydrodynamic scaling of $C_{\ell, \ell^{\prime}}(t)$ caused by the conservation of magnetization, $\left[\mathcal{H}, \sum_{\ell} S_{\ell}^{z}\right]=0$. In particular, $C_{1,1}(t) \propto t^{-\alpha}$ develops a power-law tail for times $t \gtrsim 10$ [Fig. 3(a)], while correlations $C_{\ell, 1}(t)$ build up throughout the system [cf. Fig. 1(d)], i.e., $\Sigma^{2}(t) \propto t^{\beta}$ with the spatial variance

$$
\Sigma^{2}(t)=\sum_{\ell} \ell^{2} \tilde{C}_{\ell, 1}(t)-\left[\sum_{\ell} \ell \tilde{C}_{\ell, 1}(t)\right]^{2}
$$

where $\tilde{C}_{\ell, 1}(t)=C_{\ell, 1}(t) / \sum_{\ell=1}^{L} C_{\ell, 1}(t)$ with $\sum_{\ell} \tilde{C}_{\ell, 1}(t)=1$. In Figs. 3(b) and 3(c), the impact of the Trotter step $\delta t$ on the instantaneous power-law exponents $\alpha(t)$ and $\beta(t)$ is studied for times $10 \leq t \leq 20$,

$$
\alpha(t)=-\frac{d \ln C_{1,1}(t)}{d \ln t}, \quad \beta(t)=\frac{d \ln \Sigma^{2}(t)}{d \ln t} .
$$

We find that $\alpha(t)$ exhibits damped oscillations (presumably caused by the integrability of $\mathcal{H}[76]$ ) around the mean value 
$\alpha \approx 2 / 3$, which signals superdiffusion and is consistent with a description of spin transport in terms of the Kardar-ParisiZhang (KPZ) universality class for the integrable and isotropic Heisenberg chain [29,76-81]. Remarkably, $\alpha(t)$ is essentially independent of $\delta t$ and $\alpha \approx 2 / 3$ can be readily extracted even for the largest $\delta t=2$. Likewise, $\beta(t)$ is found to remain stable up to $\delta t \leq 1$, albeit visible deviations now appear for $\delta t=2$, which is explainable by the fact that $\beta(t)$ depends on the accuracy of the Trotter decomposition on the full system while $\alpha(t)$ is a local probe. Overall, the robustness of $C_{\ell, \ell^{\prime}}(t)$ with respect to $\delta t$ is an important result and opens the door to reach nontrivial timescales with a manageable number of gates. For instance, fixing $\delta t=1$, an evolution of $L=25$ qubits up to $t=20$ requires 2400 one-qubit and 1440 two-qubit gates in our case [42].

Impact of noise.-To model the impact of erroneous gates, we consider a depolarization model with quantum channels $\mathcal{E}_{\ell}\left(\mathcal{E}_{\ell, \ell^{\prime}}\right)$ being applied after each one-qubit (twoqubit) gate [26],

$$
\begin{gathered}
\mathcal{E}_{\ell}(\rho)=\left(1-p_{1}\right) \rho+\frac{p_{1}}{3} \sum_{\mu \neq 0} \sigma_{\ell}^{\mu} \rho \sigma_{\ell}^{\mu}, \\
\mathcal{E}_{\ell, \ell^{\prime}}(\rho)=\left(1-p_{2}\right) \rho+\frac{p_{2}}{15} \sum_{(\mu, \nu) \neq(0,0)} \sigma_{\ell}^{\mu} \sigma_{\ell^{\prime}}^{\nu} \rho \sigma_{\ell}^{\mu} \sigma_{\ell^{\prime}}^{\nu},
\end{gathered}
$$

where $\rho$ is the system's density matrix; $\sigma_{\ell}^{\mu}$ with $\mu=1,2,3$ are Pauli matrices; $\sigma_{\ell}^{0}=\mathbb{1}$; and $p_{1}\left(p_{2}\right)$ are the one-qubit (two-qubit) error rates. We evaluate Eqs. (7) and (8) by averaging over quantum trajectories [26,82], $\rho(t) \approx\left(1 / N_{\mathbf{r}}\right) \sum_{\mathbf{r}}\left|\psi_{\mathcal{R}, \ell^{\prime}}^{\mathbf{r}}(t)\right\rangle\left\langle\psi_{\mathcal{R}, \ell^{\prime}}^{\mathbf{r}}(t)\right|$, where each trajectory $\left|\psi_{\mathcal{R}, \ell^{\prime}}^{\mathbf{r}}(t)\right\rangle$ corresponds to a particular history of oneand two-qubit Pauli errors. In Fig. 4, we analyze the dynamics of $C_{\ell, \ell^{\prime}}(t)$ obtained for a fixed time step $\delta t=1$ and varying error rates $p=p_{2}=10 p_{1}$. First, we consider errors only within $\mathcal{R}$ and find that they have no effect on the

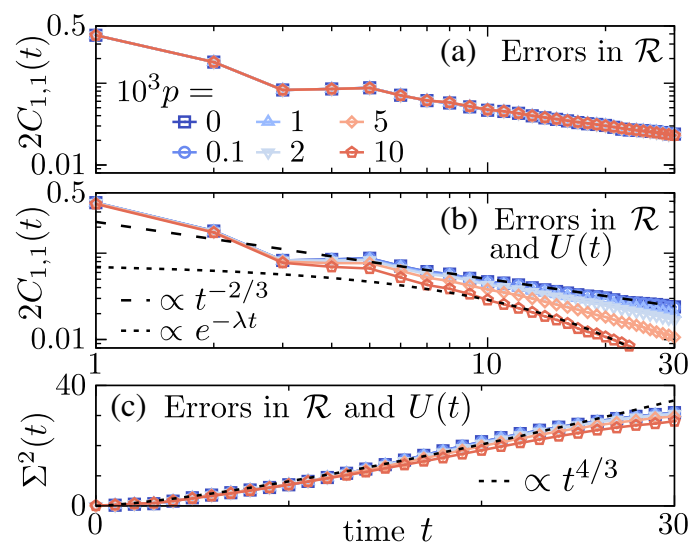

FIG. 4. Impact of errors on (a),(b) $C_{1,1}(t)$ and (c) $\Sigma^{2}(t)$. In (a) errors are present only within $\mathcal{R}$. Data are obtained for $L=20$ and $d=20$, averaged over $N_{r}=4500$ trajectories for a fixed design of $\mathcal{R}$ [42]. equal-site correlator $C_{1,1}(t)$ [Fig. 4(a)]. This exemplifies that typicality can also hold for states $\left|\psi_{\mathcal{R}, \ell^{\prime}}\right\rangle$ with nonGaussian distributions of the coefficients $c_{k}$ in the computational basis [58]. Specifically, the distribution of $p_{k}=\left|c_{k}\right|^{2}$ drifts from exponential to uniform for large error rates $[23,42]$. While this has been problematic for the sampling task in [24], it is irrelevant for our approach.

In contrast, if errors are present in both $\mathcal{R}$ and $U(t)$ [Fig. 4(b)], the decay of $C_{1,1}(t)$ depends on $p$. While a power-law tail $C_{1,1}(t) \propto t^{-\alpha}$ with $\alpha=2 / 3$ can still be extracted for $p \lesssim 2 \times 10^{-3}$ (roughly one order of magnitude smaller than currently achievable [24]), the depolarization errors cause $C_{\ell, \ell^{\prime}}(t)$ to decay exponentially for larger $p$ [26]. Compared to the local probe $C_{1,1}(t)$, the spatial variance $\Sigma^{2}(t)$ appears to be less sensitive to noise [see Fig. 4(c)] and exhibits a power-law growth even for $p=10^{-2}$. The robustness of $\Sigma^{2}(t)$ might be explained by the fact that $\left|\psi_{\mathcal{R}, \ell^{\prime}}\right\rangle$ is random and structureless at short times except for sites close to $\ell^{\prime}$. Thus, errors away from $\ell^{\prime}$ do not drastically alter the spreading of $C_{\ell, \ell^{\prime}}(t)$ and the growth of $\Sigma^{2}(t)$. This is another result of this Letter. Given the robustness of $\Sigma^{2}(t)$ [and $C_{\ell, \ell^{\prime}}(t)$ ] against Trotter and gate errors as well as the gradual improvement of technology, we expect near-term NISQ devices to provide a useful platform to extract transport coefficients, such as diffusion constants, of many-body quantum systems. In this context, the signal-to-noise ratio of the data in actual experiments can be systematically improved by increasing the number of repetitions [24,26,42].

Dynamics of $2 D$ systems. - Our approach is neither restricted to the dynamics of 1D systems nor to the choice of $\ell^{\prime}=1$. In Fig. 5(a), we repeat our analysis of the $\delta t$ dependence for a 2D Heisenberg model with $L=L_{x} \times L_{y}=25$ and choose the reference site $\ell^{\prime}=L / 2$ as the central site of the lattice. Analogous to the 1D case, we find that $C_{L / 2, L / 2}(t)$ is remarkably robust with respect to $\delta t$, with a stable hydrodynamic tail $C_{L / 2, L / 2}(t) \propto t^{-1}$, which signals the onset of conventional diffusion in $2 \mathrm{D}$ consistent with the transition from integrability to nonintegrability of $\mathcal{H}$ from 1D to 2D [29]. Finally, let us consider the state $\left|\psi_{X}\right\rangle=|\rightarrow|^{\otimes L / 2-1} \otimes|\uparrow\rangle \otimes|\rightarrow\rangle^{\otimes L / 2}$, i.e., a nonrandom

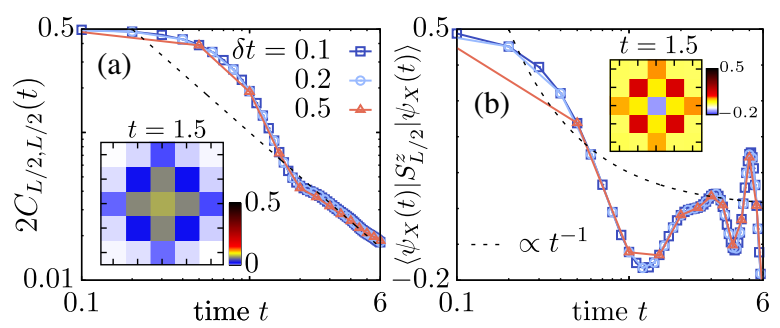

FIG. 5. Dynamics in 2D. (a) $C_{L / 2, L / 2}(t)$ for varying $\delta t$. Dashed line indicates diffusive decay $\propto t^{-1}$. Inset shows $C_{\ell, L / 2}(t)$ at $t=1.5$. (b) The nonrandom state $\left|\psi_{X}(t)\right\rangle$ (see text for details) yields dynamics incompatible with diffusion. 
product state in which spins at $\ell \neq \ell^{\prime}$ point in the $x$ direction, preparable by applying Hadamard gates on all but the central site. At $t=0$, this state yields $\left\langle\psi_{X}\left|S_{\ell}^{z}\right| \psi_{X}\right\rangle=0.5 \delta_{\ell, L / 2}$, i.e., the same as $\left|\psi_{\mathcal{R}, \ell^{\prime}}\right\rangle$. The dynamics for $t>0$ [Fig. 5(b)], however, clearly differs from $C_{L / 2, L / 2}(t)$ and is incompatible with a power-law decay. Thus, the randomness of $\left|\psi_{\mathcal{R}, \ell^{\prime}}\right\rangle$ is crucial to extract the correct hydrodynamic scaling. This is another important result.

Conclusion.-We have shown that NISQ devices provide useful platforms to simulate hydrodynamics of quantum many-body systems. Relying on random-circuit technology and "quantum parallelism," we specifically presented an efficient scheme to obtain spatiotemporal correlation functions without the need of bath or ancilla qubits. As the intrinsic accuracy of Eq. (2) improves exponentially with the number of qubits, we expect it to be scalable to larger systems. Especially for quantum many-body dynamics in $2 \mathrm{D}$, which is known to be notoriously challenging for numerical methods, simulations on NISQ devices might help to answer open questions such as the existence of many-body localization.

Recently, ergodic and nonergodic behaviors have been shown in dual-unitary circuits [83-85]. In a related work [85], Claeys and Lamacraft also consider spatiotemporal correlations such as $C_{\ell, \ell^{\prime}}(t)$. While Ref. [85] explores their dynamics for different classes of dual-unitary circuits, our work studies $C_{\ell, \ell^{\prime}}(t)$ for explicit spin systems and, moreover, highlights the usefulness of random circuits for the preparation of suitable initial states. The role of typicality in dual-unitary circuits is a question for future work.

A natural extension would be to consider thermal expectation values $\langle\bullet\rangle_{\beta}=\operatorname{Tr}\left[\bullet e^{-\beta \mathcal{H}}\right] / \operatorname{Tr}\left[e^{-\beta \mathcal{H}}\right]$ at inverse temperature $\beta$, which by virtue of typicality can be written as $\langle\bullet\rangle_{\beta} \approx\left\langle\psi_{\beta}|\cdot| \psi_{\beta}\right\rangle /\left\langle\psi_{\beta} \mid \psi_{\beta}\right\rangle$ with $\left|\psi_{\beta}\right\rangle=e^{-\beta \mathcal{H} / 2}|r\rangle$ [86], where $|r\rangle$ is a random state. While $\left|\psi_{\beta}\right\rangle$ is straightforward to compute on a classical machine, a scheme to implement the unnatural nonunitary evolution on a quantum computer has been recently proposed [87]. Thus, random circuits might also provide a means to prepare thermal states on NISQ devices, complementary to other approaches for this task [87-90].

We sincerely thank F. Barratt, J. Dborin, H. De Raedt, A. G. Green, F. Jin, and R. Steinigeweg for helpful discussions and comments. This work was funded by the European Research Council (ERC) under the European Union's Horizon 2020 research and innovation programme (Grant Agreement No. 853368).

j.richter@ucl.ac.uk

[1] R. P. Feynman, Int. J. Theor. Phys. 21, 467 (1982).

[2] U. Schollwöck, Rev. Mod. Phys. 77, 259 (2005); Ann. Phys. (Amsterdam) 326, 96 (2011).
[3] A. Weiße, G. Wellein, A. Alvermann, and H. Fehske, Rev. Mod. Phys. 78, 275 (2006).

[4] F. Verstraete, V. Murg, and J. I. Cirac, Adv. Phys. 57, 143 (2008).

[5] E. Gull, A. J. Millis, A. I. Lichtenstein, A. N. Rubtsov, M. Troyer, and P. Werner, Rev. Mod. Phys. 83, 349 (2011).

[6] H. Aoki, N. Tsuji, M. Eckstein, M. Kollar, T. Oka, and P. Werner, Rev. Mod. Phys. 86, 779 (2014).

[7] G. Carleo and M. Troyer, Science 355, 602 (2017).

[8] I. Bloch, J. Dalibard, and S. Nascimbène, Nat. Phys. 8, 267 (2012).

[9] R. Blatt and C. F. Roos, Nat. Phys. 8, 277 (2012).

[10] I. M. Georgescu, S. Ashhab, and F. Nori, Rev. Mod. Phys. 86, 153 (2014).

[11] F. Tacchino, A. Chiesa, S. Carretta, and D. Gerace, Adv. Quantum Technol. 3, 1900052 (2020).

[12] A. Kandala, A. Mezzacapo, K. Temme, M. Takita, M. Brink, J. M. Chow, and J. M. Gambetta, Nature (London) 549, 242 (2017).

[13] P. J. J. O’Malley et al., Phys. Rev. X 6, 031007 (2016).

[14] A. Chiesa, F. Tacchino, M. Grossi, P. Santini, I. Tavernelli, D. Gerace, and S. Carretta, Nat. Phys. 15, 455 (2019).

[15] A. Francis, J. K. Freericks, and A. F. Kemper, Phys. Rev. B 101, 014411 (2020).

[16] K. Choo, C. W. von Keyserlingk, N. Regnault, and T. Neupert, Phys. Rev. Lett. 121, 086808 (2018).

[17] Y. Wang, Y. Li, Z.-q. Yin, and B. Zeng, npj Quantum Inf. 4, 46 (2018).

[18] A. Smith, B. Jobst, A. G. Green, and F. Pollmann, arXiv: 1910.05351.

[19] H. Lamm and S. Lawrence, Phys. Rev. Lett. 121, 170501 (2018).

[20] A. Smith, M. S. Kim, F. Pollmann, and J. Knolle, npj Quantum Inf. 5, 106 (2019).

[21] F. Arute et al., arXiv:2010.07965.

[22] O. E. Sommer, F. Piazza, and D. J. Luitz, arXiv:2011.08853.

[23] S. Boixo, S. V. Isakov, V. N. Smelyanskiy, R. Babbush, N. Ding, Z. Jiang, M. J. Bremner, J. M. Martinis, and H. Neven, Nat. Phys. 14, 595 (2018).

[24] F. Arute et al., Nature (London) 574, 505 (2019).

[25] J. Preskill, Quantum 2, 79 (2018).

[26] M. Ippoliti, K. Kechedzhi, R. Moessner, S. L. Sondhi, and V. Khemani, arXiv:2007.11602.

[27] M. J. Gullans, S. Krastanov, D. A. Huse, L. Jiang, and S. T. Flammia, arXiv:2010.09775.

[28] P. M. Poggi, N. K. Lysne, K. W. Kuper, I. H. Deutsch, and P. S. Jessen, PRX Quantum 1, 020308 (2020).

[29] B. Bertini, F. Heidrich-Meisner, C. Karrasch, T. Prosen, R. Steinigeweg, and M. Žnidarič, Rev. Mod. Phys. 93, 025003 (2021).

[30] S. A. Wolf, D. D. Awschalom, R. A. Buhrman, J. M. Daughton, S. von Molnár, M. L. Roukes, A. Y. Chtchelkanova, and D. Treger, Science 294, 1488 (2001).

[31] L. D’Alessio, Y. Kafri, A. Polkovnikov, and M. Rigol, Adv. Phys. 65, 239 (2016).

[32] F. Borgonovi, F. M. Izrailev, L. F. Santos, and V. G. Zelevinsky, Phys. Rep. 626, 1 (2016).

[33] C. Gogolin and J. Eisert, Rep. Prog. Phys. 79, 056001 (2016). 
[34] S. Das Sarma, S. Adam, E. H. Hwang, and E. Rossi, Rev. Mod. Phys. 83, 407 (2011).

[35] C. Hess, Phys. Rep. 811, 1 (2019).

[36] A. Scheie, N. E. Sherman, M. Dupont, S. E. Nagler, M. B. Stone, G. E. Granroth, J. E. Moore, and D. A. Tennant, Nat. Phys., (2021).

[37] S. Hild, T. Fukuhara, P. Schauß, J. Zeiher, M. Knap, E. Demler, I. Bloch, and C. Gross, Phys. Rev. Lett. 113, 147205 (2014).

[38] N. Jepsen, J. Amato-Grill, I. Dimitrova, W. W. Ho, E. Demler, and W. Ketterle, Nature (London) 588, 403 (2020).

[39] T. Rakovszky, C. W. von Keyserlingk, and F. Pollmann, arXiv:2004.05177.

[40] V. Khemani, A. Vishwanath, and D. A. Huse, Phys. Rev. X 8, 031057 (2018).

[41] H. De Raedt, A. H. Hams, K. Michielsen, S. Miyashita, and K. Saito, Prog. Theor. Phys. Suppl. 138, 489 (2000).

[42] See Supplemental Material at http://link.aps.org/ supplemental/10.1103/PhysRevLett.126.230501 for a derivation of Eq. (2), details on the accuracy and of our approach and generalizations thereof, the impact of noise on the output probability distribution, different circuit designs, the decomposition of spin exchange terms into elementary gates, dynamics for shallower $\mathcal{R}$, and the extraction of the diffusion coefficient for a nonintegrable model.

[43] D. J. Luitz and Y. Bar Lev, Ann. Phys. (Amsterdam) 529, 1600350 (2017).

[44] S. Popescu, A. J. Short, and A. Winter, Nat. Phys. 2, 754 (2006).

[45] S. Goldstein, J. L. Lebowitz, R. Tumulka, and N. Zanghì, Phys. Rev. Lett. 96, 050403 (2006).

[46] P. Reimann, Phys. Rev. Lett. 99, 160404 (2007).

[47] S. Lloyd, Ph.D. Thesis, The Rockefeller University, 1988, arXiv:1307.0378.

[48] J. Gemmer, M. Michel, and G. Mahler, Quantum Thermodynamics (Springer, Berlin, 2004), https://www.doi.org/10 .1007/978-3-540-70510-9.

[49] C. Bartsch and J. Gemmer, Phys. Rev. Lett. 102, 110403 (2009).

[50] T. Heitmann, J. Richter, D. Schubert, and R. Steinigeweg, Z. Naturforsch. A 75, 421 (2020).

[51] A. Hams and H. De Raedt, Phys. Rev. E 62, 4365 (2000).

[52] T. Iitaka and T. Ebisuzaki, Phys. Rev. Lett. 90, 047203 (2003).

[53] G. A. Álvarez, E. P. Danieli, P. R. Levstein, and H. M. Pastawski, Phys. Rev. Lett. 101, 120503 (2008).

[54] T. A. Elsayed and B. V. Fine, Phys. Rev. Lett. 110, 070404 (2013).

[55] T. Monnai and A. Sugita, J. Phys. Soc. Jpn. 83, 094001 (2014).

[56] R. Steinigeweg, J. Gemmer, and W. Brenig, Phys. Rev. Lett. 112, 120601 (2014).

[57] J. Richter and R. Steinigeweg, Phys. Rev. B 99, 094419 (2019).

[58] F. Jin, D. Willsch, M. Willsch, H. Lagemann, K. Michielsen, and H. De Raedt, J. Phys. Soc. Jpn. 90, 012001 (2021).

[59] J. Richter, F. Jin, L. Knipschild, J. Herbrych, H. De Raedt, K. Michielsen, J. Gemmer, and R. Steinigeweg, Phys. Rev. B 99, 144422 (2019).
[60] B. M. Terhal and D. P. DiVincenzo, Phys. Rev. A 61, 022301 (2000).

[61] R. Somma, G. Ortiz, J. E. Gubernatis, E. Knill, and R. Laflamme, Phys. Rev. A 65, 042323 (2002).

[62] J. S. Pedernales, R. Di Candia, I. L. Egusquiza, J. Casanova, and E. Solano, Phys. Rev. Lett. 113, 020505 (2014).

[63] M. L. Baez, M. Goihl, J. Haferkamp, J. Bermejo-Vega, M. Gluza, and J. Eisert, Proc. Natl. Acad. Sci. U.S.A. 117, 26123 (2020).

[64] J. Schliemann, A. V. Khaetskii, and D. Loss, Phys. Rev. B 66, 245303 (2002).

[65] J. Emerson, Y. S. Weinstein, M. Saraceno, S. Lloyd, and D. G. Cory, Science 302, 2098 (2003).

[66] R. Oliveira, O. C. O. Dahlsten, and M. B. Plenio, Phys. Rev. Lett. 98, 130502 (2007).

[67] D. Poulin, A. Qarry, R. Somma, and F. Verstraete, Phys. Rev. Lett. 106, 170501 (2011).

[68] R. Steinigeweg, F. Heidrich-Meisner, J. Gemmer, K. Michielsen, and H. De Raedt, Phys. Rev. B 90, 094417 (2014).

[69] P. de Vries and H. De Raedt, Phys. Rev. B 47, 7929 (1993).

[70] F. Vatan and C. Williams, Phys. Rev. A 69, 032315 (2004).

[71] D. N. Page, Phys. Rev. Lett. 71, 1291 (1993).

[72] M. Žnidarič, Phys. Rev. A 76, 012318 (2007).

[73] Y. S. Weinstein, W. G. Brown, and L. Viola, Phys. Rev. A 78, 052332 (2008).

[74] M. Heyl, P. Hauke, and P. Zoller, Sci. Adv. 5, eaau8342 (2019).

[75] L. M. Sieberer, T. Olsacher, A. Elben, M. Heyl, P. Hauke, F. Haake, and P. Zoller, npj Quantum Inf. 5, 78 (2019).

[76] S. Gopalakrishnan, R. Vasseur, and B. Ware, Proc. Natl. Acad. Sci. U.S.A. 116, 16250 (2019).

[77] M. Ljubotina, M. Žnidarič, and T. Prosen, Nat. Commun. 8, 16117 (2017).

[78] M. Ljubotina, M. Žnidarič, and T. Prosen, Phys. Rev. Lett. 122, 210602 (2019).

[79] S. Gopalakrishnan and R. Vasseur, Phys. Rev. Lett. 122, 127202 (2019).

[80] J. De Nardis, M. Medenjak, C. Karrasch, and E. Ilievski, Phys. Rev. Lett. 123, 186601 (2019).

[81] F. Weiner, P. Schmitteckert, S. Bera, and F. Evers, Phys. Rev. B 101, 045115 (2020).

[82] J. Dalibard, Y. Castin, and K. Mølmer, Phys. Rev. Lett. 68, 580 (1992).

[83] B. Bertini, P. Kos, and T. Prosen, Phys. Rev. X 9, 021033 (2019).

[84] B. Bertini, P. Kos, and T. Prosen, Phys. Rev. Lett. 123, 210601 (2019).

[85] P. W. Claeys and A. Lamacraft, Phys. Rev. Lett. 126, 100603 (2021).

[86] S. Sugiura and A. Shimizu, Phys. Rev. Lett. 111, 010401 (2013).

[87] M. Motta, C. Sun, A. T. K. Tan, M. J. O’Rourke, E. Ye, A. J. Minnich, and F. G. S. L. Brandão, and G. K.-L. Chan, Nat. Phys. 16, 205 (2020).

[88] K. Temme, T. J. Osborne, K. G. Vollbrecht, D. Poulin, and F. Verstraete, Nature (London) 471, 87 (2011).

[89] J. Cohn, F. Yang, K. Najafi, B. Jones, and J. K. Freericks, Phys. Rev. A 102, 022622 (2020).

[90] S. Lu, M. C. Bañuls, and J. I. Cirac, PRX Quantum 2, 020321 (2021). 